\title{
Estado nutricional de industriários da área metropolitana do Recife e associações com fatores sociodemográficos, estilo de vida e condições de saúde
}

\author{
Nutritional status of industries in the metropolitan area of \\ Recife and associations with socio-demographic factors, \\ lifestyle and health conditions
}

Marcela Karla de Almeida Lira', Cinthia Katiane Martins Calado', Maria Goretti Pessoa de Araújo Burgos ${ }^{2}$

${ }^{7}$ Nutricionista pela Universidade Federal de Pernambuco

${ }^{2}$ Nutricionista, Professora Universidade Federal de Pernambuco

E-mail para correspondência: Maria Goretti Pessoa de Araújo Burgos gburgos@hotlink.com.br

\begin{abstract}
Resumo
Objetivo: avaliar o estado nutricional de industriários e associações com fatores sociodemográficos, estilo de vida e condições de saúde. Método: Estudo transversal realizado em uma indústria gráfica da área metropolitana do Recife, $\mathrm{PE}$, no período de maio a junho de 2011; com 146 adultos (97 homens e 49 mulheres). Os dados avaliados foram: sociodemográficos (sexo, idade, estado civil, renda, escolaridade), estilo de vida (atividade física), antropométricos (peso, estatura, circunferência da cintura, circunferência do quadril) e comorbidades. Na análise dos dados utilizouse o Teste Exato de Fisher, para associações das diferentes faixas de IMC com as variáveis analisadas; foi usado o nível de significância de 5\%. Resultados: A prevalência do excesso de peso foi de $56,16 \%$, no grupo total. Houve associação significativa da obesidade nos homens ( $P=0,038)$, com a faixa etária de 30-40 anos de ambos os sexos $(P=0,018)$ e nos indivíduos casados $(P<0,001)$. Naqueles com renda em torno de um salário mínimo, prevaleceu a eutrofia $(P<0,001)$. Não ocorreu interferência do estado nutricional nos demais fatores analisados. Dentre os perímetros avaliados, apenas a Relação Cintura/Estatura se mostrou elevada em $55,5 \%$ dos indivíduos obesos de ambos os sexos.Conclusão: $O$ excesso de peso foi prevalente em ambos os sexos, sendo a obesidade mais freqüente nos homens de união estável, onde o índice de massa corporal em todo grupo sofreu interferência da idade, estado civil e renda.
\end{abstract}

Palavras-chave: Estado Nutricional. Obesidade. Sobrepeso. Estilo de vida.

\begin{abstract}
Objective: To assess the nutritional status of industries and analyze its association with sociodemographic factors and lifestyle. Method: Cross-sectional study in a printing company in the metropolitan area of Recife, PE. Participants were 146 adults (97 men and 49 women). Sociodemographic (gender, age, marital status, income, education), lifestyle (physical activity) anthropometric (weight, height, waist circumference, hip circumference) and prevalent diseases data were
\end{abstract}


Marcela Karla de Almeida Lira, Cinthia Katiane Martins Calado, Maria Goretti Pessoa de Araújo Burgos | Estado nutricional de industriários da área metropolitana do Recife e associações com fatores sociodemográficos, estilo de vida e condições de saúde

collected. In association data we used the Fisher Exact Test. Results: The prevalence of obesity and overweight was $56.16 \%$ and underweight at only $1.3 \%$ of the sample. There was a significant association between obesity in men $(P=$ $0.038)$, aged 30-40 years of both sexes $(P=0.018)$ and married $(P<0.001)$. Those with income around one minimum wage prevailed eutrophic $(P<0.001)$. There was no interference with the nutritional status of the other factors analyzed. Among the circumferences assessed only waist ratio stature showed high obesity in 55.5 $\%$ of the group. Conclusion: Overweight was prevalent in both sexes, being more frequent in men of stable marriage where the body mass index in every group suffered interference of age, marital status and income.

Keywords: Nutritional Status. Obesity. Overweight. Lifestyle.

\section{INTRODUÇÃO}

Um estado nutricional adequado é uma das formas de manter boa saúde e consequentemente aumentar a produtividade de industriários, que trabalhem em empresas de qualquer ramo. Neste aspecto, a avaliação nutricional sistemática propõe estratégias para tomada de decisão dentro do planejamento alimentar institucional ${ }^{1}$.

Desde a década de 1960, a Organização Mundial de Saúde (OMS) propõe instrumentos de avaliação nutricional para detectar precocemente os problemas nutricionais mais prevalentes em cada país e dessa forma, estabelecer medida de prevenção e controle ${ }^{2}$.

A sociedade moderna tem vivenciado, nas últimas décadas, um dinâmico e complexo processo de mudanças nos padrões alimentares e nutricionais, nos perfis demográfico, socioeconômico e epidemiológico. Tal processo vem acontecendo de maneira diferenciada em países, regiões e grupos sociais diversos ${ }^{3,4}$.

Nos últimos anos o sobrepeso e a obesidade aumentaram consideravelmente e, são globalmente responsáveis por $44 \%$ da carga global de diabetes mellitus tipo 2 (DM2), 23\% de doença isquêmica do coração e entre 7\% e 41\% para alguns tipos de câncer, contribuindo para morbimortalidade da população ${ }^{5,6}$. No Brasil, as doenças cardiovasculares estão nas estatísticas de saúde como a primeira causa de morte7.

Os hábitos alimentares e a prática de atividades físicas exercem influência sobre o balanço energético, sendo considerados os principais fatores, passíveis de modificação, determinantes da obesidade ${ }^{8}$. 
Marcela Karla de Almeida Lira, Cinthia Katiane Martins Calado, Maria Goretti Pessoa de Araújo Burgos| Estado nutricional de industriários da área metropolitana do Recife e associações com fatores sociodemog ráficos, estilo de vida e condições de saúde

Estudos locais, envolvendo grupos populacionais menores e definidos, são úteis para o estabelecimento de valores de referência regionais e para a comparação com outros grupos de características similares. Estas razões motivaram a realização do presente trabalho, por ser $01^{\circ}$ realizado no Estado, em funcionários de uma empresa privada de pequeno porte, da área metropolitana de Recife, Pernambuco.

Assim, é objetivo deste trabalho verificar o estado nutricional de industriários e analisar sua associação com fatores sócio-demográficos, estilo de vida e condições de saúde.

\section{MÉTODO}

Trata-se de um estudo transversal, realizado com funcionários de uma indústria gráfica, localizada no município do Cabo de Santo Agostinho, no estado de Pernambuco no segundo semestre de 2011. A pesquisa foi aprovada pelo Comitê de Ética em Pesquisa em Seres Humanos do Instituto de Medicina Integral Professor Fernando Figueira - IMIP, ( $\mathrm{n}^{0}$ 1.850). Todos os participantes assinaram um termo de consentimento livre e esclarecido antes da coleta de dados. Foram critérios de inclusão ter idade de 20-59 anos, estar trabalhando e realizando almoço na empresa no último ano, sem licenças médicas por mais de 2 dias e estar no último ano no mesmo turno de trabalho. Foram excluídos aqueles com obesidade mórbida, que realizaram cirurgias plásticas ou de grande porte, portadores de doenças que promovam redistribuição de gordura ou retenção hídrica em qualquer nível.

Desta maneira, foram analisados adultos, dos setores operacional, administrativo, turnos diurno e noturno. Foram coletados dados sociodemográficos (sexo, idade, escolaridade, estado civil e renda), antropométricos (massa corporal, estatura, perímetro da cintura e do quadril), além de informações sobre nível de atividade física (tipo, frequência e tempo), doenças prevalentes e uso de medicação. Para a obtenção dos dados antropométricos utilizou-se uma balança eletrônica portátil, da marca Lifetime Lithium Power, com capacidade de 150 kg, modelo MEA - 03200. Para a obtenção da estatura foi utilizado uma fita métrica inelástica com sensibilidade de $0,1 \mathrm{~cm}$, fixada em uma parede plana e com o auxílio de um esquadro. A técnica para a coleta dessas medidas seguiu recomendação de Lohmann et al` .

O estado nutricional dos indivíduos foi classificado segundo o índice de massa corporal - IMC (peso em kg dividido pela estatura em metros ao quadrado), segundo critérios estabelecidos pela Organização Mundial da Saúde ${ }^{8}$ (IMC $<18,5 \mathrm{~kg} / \mathrm{m}^{2}$. - 
Marcela Karla de Almeida Lira, Cinthia Katiane Martins Calado, Maria Goretti Pessoa de Araújo Burgos| Estado nutricional de industriários da área metropolitana do Recife e associações com fatores sociodemog ráficos, estilo de vida e condições de saúde

baixo peso; 18,5 a $24,9 \mathrm{~kg} / \mathrm{m}^{2}$. - eutrofia; 25,0 a $29,9 \mathrm{~kg} / \mathrm{m}^{2}$. - sobrepeso; e valores de $\mathrm{IMC}>30,0 \mathrm{~kg} / \mathrm{m}^{2}$. - obesidade). $\mathrm{O}$ termo excesso de peso foi utilizado para agrupamento formado pelos indivíduos como sobrepeso ou obesidade, ou seja, indivíduos com IMC $\geq 25 \mathrm{~kg} / \mathrm{m}^{2}$.

Para a coleta da circunferência da cintura (CC) a medida foi tomada com o indivíduo em pé, com os pés juntos, os braços estendidos lateralmente e o abdome relaxado. A medida deve ser tomada em plano horizontal com fita inelástica no ponto mais estreito do tronco ${ }^{9}$. Os pontos de corte utilizados como critério de classificação foram os sugeridos pela OMS, os quais especificam que o risco de complicações metabólicas associadas à obesidade está elevado para homens com valores $\geq 94 \mathrm{~cm}$ e muito elevado com valores $\geq 102 \mathrm{~cm}$ e para mulheres está elevado com valores $\geq 80$ cm e muito elevado com valores $\geq 88 \mathrm{~cm}$, enquanto a circunferência do quadril (CQ) foi medida na altura máxima dos glúteos, dispondo a fita antropométrica em plano horizontal, que deve ser estendida sobre a pele sem comprimir as partes moles ${ }^{8,10}$. Relação da cintura para o quadril (RCQ): é calculada dividindo a CC (cm) pela CQ $(\mathrm{cm})^{8}$. Uma RCQ de 1,0 ou mais para homens e de 0,8 ou mais para as mulheres é indicativo de obesidade androide e risco aumentado de doenças relacionadas com a obesidade $^{8}$. A relação cintura/estatura (RCE) foi obtida pelo quociente entre a CC e a estatura $(\mathrm{cm})$. A vantagem desse índice sobre o emprego isolado da cintura se faz por tratar-se de um ponto de corte único $(0,50)$, que pode ser usado em ambos os sexos, pois é ajustado pela estatura ${ }^{11}$.

As análises foram realizadas no Software STATA/SE 9.0 e no Excel 2007, adotandose um nível de significância de $\mathrm{p}<0,05$. Para verificar a existência de associação, foi utilizado o Teste Exato de Fisher para as variáveis categóricas.

\section{RESULTADOS}

A empresa possuía 184 funcionários, sendo o estudo realizado com $92,4 \%$ do quadro (146 indivíduos). A idade média de 32,3 \pm 8,5 anos, maioria do sexo masculino, 51,4\% solteiros, viúvos ou divorciados, $74 \%$ tinham renda familiar igual a 1 salário mínimo, 89,7\% apresentavam mais de 8 anos de estudo e 69,2\% eram sedentários, sendo a musculação a atividade mais frequente naqueles que não eram sedentários (Tabela 1). 
Marcela Karla de Almeida Lira, Cinthia Katiane Martins Calado, Maria Goretti Pessoa de Araújo Burgos | Estado nutricional de industriários da área metropolitana do Recife e associações com fatores sociodemog ráficos, estilo de vida e condições de saúde

Tabela 1 - Características sociodemográficas e de estilo de vida de funcionários de uma indústria gráfica, Cabo de Santo Agostinho (PE), 2011.

\begin{tabular}{lcc}
\hline Variáveis & N & $\%$ \\
\hline Sexo & 49 & 33,6 \\
Feminino & 97 & 66,4 \\
Masculino & & \\
Idade & 60 & 41,1 \\
$<30$ & 57 & 39,0 \\
$30 \mid$ - 40 & 29 & 19,9 \\
$\geq 40$ & & \\
Renda & 108 & 74,0 \\
1-SM* & 23 & 15,8 \\
2- 3 SM & 15 & 10,2 \\
4-5 SM & 15 & 10,3 \\
Escolaridadet & 131 & 89,7 \\
Até 8 anos & & \\
Mais de 8 anos & 45 & 30,8 \\
Atividade Física & 101 & 69,2 \\
Sim & & \\
Não & 10 & 22,2 \\
Tipo de Atividade Física & 18 & 40,0 \\
Caminhada & 1 & 2,2 \\
Musculação & 9 & 8,9 \\
Alongamento & 4 & 6,7 \\
Futebol & 3 & \\
Bicicleta & & \\
Corrida & & \\
\hline
\end{tabular}

*SM:Salário Mínimo; †Escolaridade:anos de estudo concluídos.

A análise do estado nutricional detectou 42,5\% de eutróficos e 56,16\% de excesso de peso (Tabela 2). As proporções de risco de doenças cardiovasculares apresentaram percentuais distintos, de acordo com os parâmetros utilizados, com variação de $24 \%$ a 55\%, com destaque para a relação Cintura/Quadril (Tabela 2).

Tabela 2 - Classificação do estado nutricional de funcionários de uma indústria gráfica, Cabo de Santo Agostinho (PE), 2011.

\begin{tabular}{lcc}
\hline Variáveis & N & $\%$ \\
\hline Índice de Massa Corporal* & 2 & \\
Baixo Peso & 62 & 4,3 \\
Adequado & 56 & 38,5 \\
Sobrepeso & 26 & 17,8 \\
Obeso & 55 & 37,6 \\
Perímetro da Cinturat & 91 & 62,4 \\
InadequadoAdequado & & \\
Relação Cintura/Quadril ¥, & 35 & 24,0 \\
Inadequado & 111 & 76,0 \\
Adequado & 81 & 55,5 \\
Relação Cintura/estatura§ & 65 & 44,5 \\
Inadequado/Adequado &
\end{tabular}

*Classificação Organização Mundial de Saúde (OMS), 1998 para o Índice de Massa Corporal ( $\left.\mathrm{kg} / \mathrm{m}^{2}\right) ; \mathrm{IMC}<18,5$ - baixo peso; 18,5 a 24,9 - adequado; 25,0 a 29,9 - sobrepeso; e IMC > 30,0 - obesidade. † Homens $\geq 94 \mathrm{~cm}$ e mulheres $\geq 80 \mathrm{~cm}$ (WHO, 1998). $\neq$ Homens $\geq 1.0$ e mulheres $\geq 0,8(\mathrm{WHO}, 1995)$. 8 Homens e mulheres $\geq 0,50$ (Ashwell, 2005)

Foi detectado maior sobrepeso nas faixas etárias de 30 a 40 anos, entre os casados, que também apresentaram maior prevalência de obesidade. 
Marcela Karla de Almeida Lira, Cinthia Katiane Martins Calado, Maria Goretti Pessoa de Araújo Burgos | Estado nutricional de industriários da área metropolitana do Recife e associações com fatores sociodemográficos, estilo de vida e condições de saúde

Na tabela 3 verificam-se variáveis socioeconômicas associadas ao estado nutricional, com associação significativa entre idade, sexo, estado civil e IMC.

Foi detectada reduzida frequência de patologias crônicas $(7,5 \%)$, como diabetes e hipertensão, além de baixo uso de medicações contínuas (5,5\%). Estas variáveis não mostraram associações com o IMC.

Tabela 3 - Prevalência do estado nutricional de acordo com o sexo, características socioeconômicas, presença de doenças, uso de medicações e atividade física de industriários, Cabo de Santo Agostinho (PE), 2011.

\begin{tabular}{|c|c|c|c|c|c|}
\hline \multirow[b]{2}{*}{ Variáveis } & \multicolumn{5}{|c|}{ IMC } \\
\hline & $\begin{array}{l}\leq 18,5 \\
n(\%)\end{array}$ & $\begin{array}{c}18,5 \text { I- 24,9 } \\
\text { n (\%) } \\
\end{array}$ & $\begin{array}{c}25,0 \text { |- 29,9 } \\
\text { n (\%) }\end{array}$ & $\begin{array}{l}\geq 30,0 \\
n(\%)\end{array}$ & p-valor * \\
\hline \multicolumn{6}{|l|}{ Sexo } \\
\hline Feminino & $2(100,0)$ & $17(27,4)$ & $24(42,9)$ & $6(23,1)$ & 0,038 \\
\hline Masculino & $0(0,0)$ & $45(72,6)$ & $32(57,1)$ & $20(76,9)$ & \\
\hline \multicolumn{6}{|l|}{ Idade } \\
\hline$<30$ & $1(50,0)$ & $34(54,9)$ & $20(35,7)$ & $5(19,2)$ & 0,018 \\
\hline $30 \mid-40$ & $0(0,0)$ & $18(29,0)$ & $26(46,4)$ & $13(50,0)$ & \\
\hline$\geq 40$ & $1(50,0)$ & $10(16,1)$ & $10(17,9)$ & $8(30,8)$ & \\
\hline \multicolumn{6}{|l|}{ Estado Civil } \\
\hline Solteiro/Viúvo/Divorciado & $2(100,0)$ & $40(64,5)$ & $24(42,9)$ & $9(34,6)$ & $<0,001$ \\
\hline Casado & $0(0,0)$ & $22(35,5)$ & $32(57,1)$ & $17(65,4)$ & \\
\hline \multicolumn{6}{|l|}{ Renda } \\
\hline $1 \mathrm{SM}+$ & $1(50,0)$ & $55(88,7)$ & $41(73,2)$ & $11(42,3)$ & $<0,001$ \\
\hline $2-3 S M$ & $0(0,0)$ & $6(9,7)$ & $8(14,3)$ & $9(34,6)$ & \\
\hline $4-5 \mathrm{SM}$ & $1(50,0)$ & $1(1,6)$ & $7(12,5)$ & $6(23,1)$ & \\
\hline \multicolumn{6}{|l|}{ Escolaridade } \\
\hline Até 8 anos & $0(0,0)$ & $10(16,1)$ & $3(5,4)$ & $2(7,7)$ & 0,277 \\
\hline Mais de 8 anos & $2(100,0)$ & $52(83,9)$ & $53(94,6)$ & $24(92,3)$ & \\
\hline \multicolumn{6}{|l|}{ DM § } \\
\hline Sim & $0(0,0)$ & $1(1,6)$ & $0(0,0)$ & $1(3,8)$ & 0,493 \\
\hline Não & $2(100,0)$ & $61(98,4)$ & $56(100,0)$ & $25(96,2)$ & \\
\hline \multicolumn{6}{|l|}{ HAS } \\
\hline Sim & $\mathrm{O}(0,0)$ & $\mathrm{O}(0,0)$ & $\mathrm{O}(0,0)$ & $1(3,8)$ & 0,192 \\
\hline Não & $2(100,0)$ & $62(100,0)$ & $56(100,0)$ & $25(96,2)$ & \\
\hline \multicolumn{6}{|c|}{$\begin{array}{l}\text { Medicações } \\
\text { hipotensoras/hipoglicemiante }\end{array}$} \\
\hline Sim & $\mathrm{O}(0,0)$ & $3(4,8)$ & $2(3,6)$ & $3(11,5)$ & 0,421 \\
\hline Não & $2(100,0)$ & $59(95,2)$ & $54(96,4)$ & $23(88,5)$ & \\
\hline \multicolumn{6}{|l|}{ Atividade Física } \\
\hline Sim & $0(0,0)$ & $20(32,3)$ & $19(33,9)$ & $6(23,1)$ & 0,678 \\
\hline Não & $2(100,0)$ & $42(67,7)$ & $37(66,1)$ & $20(76,9)$ & \\
\hline
\end{tabular}

(*) Teste Exato de Fisher; Classificação Organização Mundial de Saúde (OMS), 1998 para o Índice de Massa Corporal (kg/m²);IMC < 18,5- baixo peso; 18,5 a 24,9 - adequado; 25,0 a 29,9 - sobrepeso; e IMC > 30,0 - obesidade. † SM:Salário Mínimo; † Escolaridade: anos de estudo concluídos; $§ D M=$ Diabetes Mellitus; ||HAS = Hipertensão Arterial Sistêmica.

\section{DISCUSSÃO}

A análise dos dados antropométricos dos industriários, indicou frequência de excesso de peso, comprovando a presença da epidemia de obesidade em adultos jovens, da região Nordeste do Brasil. Enquanto 1,3\% apresentaram baixo peso, indicando baixa exposição da população à desnutrição, 56,2\% mostraram excesso de peso. Este resultado é observado e explicado por Monteiro et al., ao analisar dados de 3 pesquisas de base populacional realizadas no Brasil entre 1975 e 1997. Estes 
Marcela Karla de Almeida Lira, Cinthia Katiane Martins Calado, Maria Goretti Pessoa de Araújo Burgos| Estado nutricional de industriários da área metropolitana do Recife e associações com fatores sociodemog ráficos, estilo de vida e condições de saúde

estudos detectaram que nos adultos o sobrepeso parece estar substituindo a desnutrição como problema de saúde pública' ${ }^{12}$.

O sobrepeso e a obesidade foram mais frequentes no sexo masculino, em concordância com Schneider, et al. $^{13}$, com grupo de funcionários de uma penitenciária em São Paulo, onde encontrou excesso de peso em 78,12\% dos homens e $56,41 \%$ das mulheres. Do mesmo modo a prevalência de sobrepeso também foi maior nos homens, no estudo realizado por Sousa et al., entre funcionários de unidades de saúde do Piauí1 ${ }^{14}$. Este resultado pode ser explicado nesta faixa etária por dois fatos: 1) a maior preocupação do sexo feminino com a estética corporal, tendo em vista que nas sociedades modernas o padrão de elegância e beleza passa pela magreza $^{15}$; 2) diferenças entre homens e mulheres com relação a fatores comportamentais, como padrão de consumo alimentar, prática de atividade física, consumo de bebidas alcoólicas entre outros ${ }^{15}$. Ao mesmo tempo $\operatorname{Costa}^{16}$, estudando funcionários públicos do Rio de Janeiro encontrou níveis de obesidade maior nas mulheres, fato não constatado neste estudo provavelmente pelo reduzido grupo de mulheres na nossa amostra.

O uso das circunferências como preditores de doenças crônico-degenerativas está bem estabelecido, é possível encontrar muitos estudos que enfocam os determinantes associados a sua elevação $0^{17-19}$. Entretanto, apesar de diversos estudos relativamente recentes demonstrarem que a relação cintura/estatura é um bom discriminador de obesidade abdominal relacionada a fatores de risco cardiovascular, não encontramos estudos oriundos de amostras representativas que avaliassem os fatores associados à elevação da $\mathrm{RCE}^{20-22}$.

Oliveira et al. ${ }^{23}$, em estudo realizado com a população adulta de Salvador, encontrou que a obesidade abdominal revelou frequências de risco aumentado em $(28,1 \%)$, valor inferior ao encontrado no presente estudo (37,6\%). Porém, no estudo realizado no Maranhão ocorreu prevalência superior (46,3\%), demonstrando que o problema pode ter magnitude potencialmente mais elevada em algumas populações ${ }^{24-25}$.

A prevalência de excesso de peso entre os industriários integrantes da amostra $(56,16 \%)$ se revelou maior que a encontrada na última pesquisa de base populacional realizada no Brasil, em que se estimou para o Nordeste Urbano 38,6\% de excesso de peso. Ao mesmo tempo observou-se que as proporções de sobrepeso e obesidade aumentaram na faixa etária de 30 a 40 anos, estando em concordância a POF 20022003, em que a relação da obesidade com a idade reproduz, em parte, o padrão para 
Marcela Karla de Almeida Lira, Cinthia Katiane Martins Calado, Maria Goretti Pessoa de Araújo Burgos| Estado nutricional de industriários da área metropolitana do Recife e associações com fatores sociodemog ráficos, estilo de vida e condições de saúde

o excesso de peso, o qual tende a aumentar com a idade, sendo as maiores prevalências encontradas acima dos 35 anos em ambos os sexos ${ }^{25}$. Francischi et al. ${ }^{26}$, justificam o ganho de peso com o envelhecimento em razão de fatores como declínio na taxa de metabolismo basal, em consequência da perda de massa muscular, diminuição da prática de atividade física e aumento no consumo alimentar.

Neste estudo, a prevalência de sobrepeso e obesidade foi maior entre aqueles que referiram ter união conjugal estável. Castanheira et al. ${ }^{18}$, em estudo realizado no Rio Grande do Sul, encontraram que homens e mulheres vivendo com companheiro(a) apresentaram maior perímetro abdominal. Em concordância, Teachman et al. ${ }^{27}$, encontrou que os casados tendem mais a obesidade e sobrepeso do que os entrevistados nunca casados ou divorciados, independente do sexo.

Por sua vez, Teichmann et al. ${ }^{28}$, identificaram a prevalência de fatores de risco associados à pré-obesidade e obesidade em 1.358 mulheres adultas, no Rio Grande do Sul, com maior prevalência de pré-obesidade entre as mulheres casadas/união conjugal estável $(44,5 \%)$. Outro estudo, conduzido, por Hajian et al. ${ }^{29}$, no Irã com adultos de 20 a 70 anos de idade, também mostrou associação entre casamento e risco aumentado de obesidade. Gigante et al..$^{30}$, por meio de dados secundários do VIGITEL 2006, apontaram maior prevalência de obesidade e excesso de peso para ambos os sexos com união estável.

Holanda et al. ${ }^{31}$, em estudo realizado com adultos de Teresina, observaram que a prevalência de sobrepeso e obesidade identificada tanto pelo IMC quanto pela medida da CC, foi maior entre aqueles que possuíam união estável, sem diferenças entre os sexos. A influência da condição conjugal sobre o estado nutricional não está esclarecida, pois em alguns estudos, como o de Sarturi et al.32, não foram encontradas evidências de relação positiva entre essas variáveis.

Quando se estuda a estratificação por renda, observa-se maior prevalência de excesso de peso entre os industriários de menor renda. Resultados também encontrados no Brasil por Monteiro et al., entre famílias de mais baixa renda. Diferentemente dos nossos achados, alguns autores relataram um incremento de sobrepeso e obesidade com o aumento de renda para os homens ${ }^{14,17,36}$.

Nos países desenvolvidos a obesidade tende a ser mais frequente entre indivíduos de menor renda. Para os países em desenvolvimento, existe uma tendência inversa, ou seja, maior frequência de obesidade nos estratos de melhor nível socioeconômico ${ }^{12-}$ 33 . 
Com relação à variável escolaridade, no presente estudo, foi observado que o excesso de peso apresentou associação positiva. Não concordando com os achados de Peixoto et $\mathrm{al}^{36}$, que detectaram maior prevalência de excesso de peso em mulheres com até quatro anos de estudo (47,8\%); para os homens, obtiveram maior prevalência de excesso de peso $(52,2 \%)$ entre aqueles com maior escolaridade $\geq 12$ anos de estudo, semelhantes aos dados desta amostra. No entanto, no estudo de Holanda et al. ${ }^{31}$, realizado em Teresina, foi observado associação inversa entre excesso de peso e escolaridade entre as mulheres, porém o grau de escolaridade não esteve associado com o excesso de peso entre os homens. Diante de resultados diferentes na literatura, ainda não é consenso entre os pesquisadores que o baixo nível de escolaridade seja um fator de risco para a obesidade.

Entre os industriários desta pesquisa as proporções de excesso de peso definidas através do IMC foram elevadas principalmente em homens, na medida em que representa uma elevação do risco de morbidade, invalidez e mortalidade, com enorme custo social. As implicações do aumento da prevalência da obesidade observadas em homens e mulheres são de grande amplitude para a saúde pública no Brasil. Um ganho de peso na vida adulta de 5\% em relação ao peso referido aos 20 anos de vida está sendo relacionado à maior ocorrência de hipertensão (HAS), dislipidemia e hiperinsulinemia ${ }^{14,37}$.

Quanto à atividade física, apesar de não ter sido encontrada associação entre prática regular de exercício físico com excesso peso no grupo, o elevado número de sedentarismo encontrado, corrobora com achados de Nascimento e Mendes, estes observaram que mais da metade dos funcionários de um centro de Saúde-Escola de Ribeirão Preto não praticava atividade física. Do mesmo modo Castro et al., constataram em pesquisa, que mais da metade de funcionários do sexo masculino de uma empresa metalúrgica do Rio de Janeiro, que apresentava excesso de peso, não praticavam atividade física de lazer ${ }^{38,39}$.

Por escassez de dados na literatura ficamos impossibilitados de discutirmos os resultados relacionados à presença de patologias e uso de medicações.

\section{CONCLUSÃO}

A análise do estado nutricional dos industriários participantes deste estudo confirmou que o aumento prevalência de excesso de peso é um dos graves problemas de saúde pública no Brasil. Nesta amostra o excesso de peso predominou nos homens 
Marcela Karla de Almeida Lira, Cinthia Katiane Martins Calado, Maria Goretti Pessoa de Araújo Burgos | Estado nutricional de industriários da área metropolitana do Recife e associações com fatores sociodemog ráficos, estilo de vida e condições de saúde

adultos jovens, de união estável, enquanto o IMC de todo grupo sofreu interferência da idade, do estado civil e da renda. O excesso de peso em conjunto com o sedentarismo indica que ações efetivas precisam ser desenvolvidas para este grupo de trabalhadores.

\section{REFERÊNCIAS}

1. Lacey K, Pritchett E. Nutrition care process and model: ADA adopts road map to quality care and outcomes massagement. J Am Diel Assoc. 2003;103(8):1061-72.

2. Gibson R. Principles of nutritional assessment. $2^{\mathrm{a}}$ ed. New York: Oxford University Press. 2005.

3. Organização Mundial de Saúde. Dieta, nutrición y prevención de enfermidades crônicas. (Série de informestécnicos, 797). Genebra: OMS. 1990.

4. Popkin B. The nutrition transition in low-income countries: an emerging crisis. Nutr Rev.1994;52(9): 285-98.

5. World Health Organization. Global health risks: mortality and burden of disease attributable to selected major risks. Geneva: World Health Organization; 2009.

6. World Health Organization. Global status report on noncommunicable diseases 2014. Geneva: World Health Organization; 2014.

7. Ministério da Saúde. Resumo Executivo Saúde Brasil 2014: Uma análise da situação de saúde e das causas externas. Secretaria de Vigilância em Saúde Departamento de Vigilância de Doenças e Agravos não Transmissíveis e Promoção da Saúde. Brasília, DF; 2015.

8. World Health Organization. Obesity: Preventing and managing the global epidemic. Report of WHO Consultation on Obesity. Geneva: WHO; 1998.

9. Lohman TG, Roche AF, Martorell R. Antropometric standardization reference manual. Champaing (IL): Human Kinetics Books; 1988.

10. World Health Organization. Physical status: use and interpretation of anthropometry. Report of a WHO expert committee. Geneva: WHO; 1995.

11. Ashwell M, Hsieh SD. Six reasons why the waist-to-height ratio is a rapid and effective global indicator for health risks of obesity and how its use could simplify the international public health message on obesity. Int J Food SciNutr. 2005;56(5):303-7.

12. Monteiro CA, Conde WL, Popkin BM. Is obesity replacing or adding to undernutrition? Evidence from different social classes in Brazil. Public Health Nutr 2002; 5(1A):105-12.

13. Schneider VC, Carvalho SAT, Pereira CAM, Magalhães P. Avaliação antropométrica em funcionários de uma penitenciária no município de Itirapina - SP. Alim. Nutr. 2011; 22(4):593-99.

14. Sousa RMRP, Sobral DP, Paz SMRS, Martins MCC. Prevalência de sobrepeso e obesidade entre funcionários plantonistas de unidades de saúde de Teresina, Piauí. Rev. Nutr. 2007; 20(5): 473-82. 
Marcela Karla de Almeida Lira, Cinthia Katiane Martins Calado, Maria Goretti Pessoa de Araújo Burgos| Estado nutricional de industriários da área metropolitana do Recife e associações com fatores sociodemog ráficos, estilo de vida e condições de saúde

15. Silva DO. O Fiel da Balança na História do Corpo Obeso de Mulheres de Baixa Renda $\neg$ Manguinhos, Rio de Janeiro. [disertation]. Rio de Janeiro: Escola Nacional de Saúde Pública, Fundação Oswaldo Cruz. 1995.

16. Costa MAP, Vasconcelos AGG, Fonseca MJM. Prevalência de obesidade, excesso de peso e obesidade abdominal e associação com prática de atividade física em uma universidade federal. Rev Bras epidemiol. 2014;17(2): 421-36.

17. Lean MEJ, Han TS, Deurenberg P. Predicting body composition by densitometryfrom simple anthropometric measurements. Am J Clin Nutr.1996;63(1):4-14

18. Castanheira M, Olinto MTA, Gigante DP. Associação de variáveis sóciodemográficas e comportamentais com a gordura abdominal em adultos: estudo de base populacional no Sul do Brasil. Cad Saúde Pública. 2003;19(1):55-65.

19. Franque D, Witcmann FMA, Prá D. estilo de vida e fatores de risco para o sobrepeso e obesidade em mulheres de baixa renda. Cinergis 2007;8(1):40-9.

20. Haun DR, Pitanga FJG, Lessa I. Razão cintura/estatura comparado a outros indicadores antropométricos de obesidade como preditor de risco coronariano elevado. Assoc Med Bras. 2009;55(6):705-11.

21. Lin WY, Chen CY, Lo H, Hsia HH, Liu IL, Lin RS, Shau WY, Huang KC. Optimal cut-off values for obesity: using simple antropometric indices to predict cardiovascular risk factores in Taiwan. Int J Obes Relat Metab Disord 2002; 26(9):1232-8.

22. Huang KC, Lin WY, Lee LT, Chen CY, Lo H, Hsia HH, Liu LL, Shau WY, Lin RS. Four anthropometric indices and cardiovascular risk factors in Taiwan. Int J Obes Relat Metab Disord 2002;26(8):1060-8.

23. Oliveira LPM, Assis AMO, Silva MCM, Santana MLP, Santos NS, Pinheiro SMC, Barreto LM, Souza CO. Fatores associados a excesso de Peso e concentração de gordura abdominal em adultos na cidade de salvador, Bahia, Brasil. Cad Saúde Pública. 2009; 25(3):570-82.

24. Veloso HJF, Silva AUM. Prevalência e fatores associados à obesidade abdominal e ao excesso de peso em adultos maranhenses. Bras. Epidemiol 2010; 13(3):400-12.

25. Instituto Brasileiro de Geografia e Estatística. Pesquisa de orçamento familiar 2002-2003. Análise da disponibilidade domiciliar de alimentos e do estado nutricional no Brasil.IBGE, Rio de Janeiro (2004),40-76.

26. Francischi RPP, Pereira LO, Freitas CS, Klopfer ML, Santos RC, Vieira P, Junior Lancha AH. Obesidade:atualização sobre sua etiologia, morbidade e tratamento. Rev Nutr. 2000;13(1):17-29.

27. Tachman J. Body Weight, Marital Status, and Changes in Marital Status. J Fam Issues. 2016;37(1):74-96.

28. Teichmann L, Olinto MTA, Costa JSD, Ziegler D. Fatores de risco associados ao sobrepeso e a obesidade em mulheres de São Leopoldo, RS. Rev Bras Epidemiol .2006;9(6):360-73.

29. Hajian-Tilaki KO, Heidari B. Prevalence of obesity, central obesity and the associated factors in urban population aged 20-70 years, in the north of Iran: a population-based study and regression approach. Obes Ver. 2007;8:(1)3-10. 
Marcela Karla de Almeida Lira, Cinthia Katiane Martins Calado, Maria Goretti Pessoa de Araújo Burgos | Estado nutricional de industriários da área metropolitana do Recife e associações com fatores sociodemog ráficos, estilo de vida e condições de saúde

30. Gigante DP, Moura EC, Sardinha LMV. Prevalência de excesso de peso e obesidade e fatores associados, Brasil, 2006. Rev Saúde Pública. 2009; 43(2):839

31. Holanda LGM, Martins MCC, Filho MDS, Carvalho CMRG, Assis RC, Leal LMM, Mesquita LPL, Costa EM. Excesso de peso e adiposidade central em adultos de Teresina-PI. Rev Assoc Med Bras. 2011;57(1):50-55.

32. Sarturi JB, Neves J, Peres KG. Obesity in adults people: a population based study in a small town in South of Brazil, 2005. Ciênc Saúde Coletiva. 2010; 15:105-13.

33. Sichieri R, Coitinho DC, Leão MM, Recine E, Everhart JE. High temporal, geographic and income variation in body mass índex among adults in Brazil. American Journal of Public Health.1994; 84(5):793-798.

34. Sobal J, Stunkard AJ. Socioeconomics status and obesity: areview of the literature. Psychol Bull 1989; 105(2):260-75.

35. McLaren L. Socioeconomic status and Obesity. Epidemiol Rev .2007; 29: 29-48.

36. Peixoto MRG, Monego ET, Alexandre VP, Souza RGM, Moura EC. Monitoramento por entrevistas telefônicas de fatores de risco para doenças crônicas: experiência de Goiânia, Goiás, Brasil. Cad Saúde Pública. 2008; 24(6):1323-33.

37. Everson AS, Goldberg DE, Helmrich SP, Lakka TA, Lynch JW, Kaplan GA, Salonen JT. Weight gain and risk of developing Insulin Resistance Syndrome. Diabetes Care 1998; 21(10):1637-43.

38. Nascimento LC, Mendes IJM. Perfil de saúde dos trabalhadores de um centro de saúde-escola. Rev Latino-Am Enfermagem. 2002; 10(4) :502-8

39. Castro MBT, Anjos LA, Lourenço PM. Padrão dietético e estado nutricional de operários de uma empresa metalúrgica do Rio de Janeiro, Brasil. Cad Saúde Pública. 2004;20(4):926-34.

Submissão: 15/08/2019

Aprovação: 31/03/2021 